\title{
Plasma 2-hydroxyglutarate and hexanoylcarnitine levels are potential biomarkers for skeletal muscle toxicity in male Fischer 344 rats
}

\author{
Hisakuni Obayashi'1,2, Naoko Kobayashi², Yoshikazu Nezu², Takashi Yamoto², \\ Mitsuyuki Shirai ${ }^{1}$ and Fumitoshi Asai ${ }^{1}$ \\ ${ }^{1}$ Laboratory of Veterinary Pharmacology, School of Veterinary Medicine, Azabu University \\ 1-17-71 Fuchinobe, Chuo-ku, Sagamihara, Kanagawa 252-5201, Japan \\ ${ }_{2}^{2}$ Medicinal Safety Research Laboratories, Daiichi-Sankyo Co., Ltd. \\ 1-16-13 Kitakasai, Edogawa-ku, Tokyo 134-8630, Japan
}

(Received January 11, 2017; Accepted April 6, 2017)

\begin{abstract}
To identify new candidate biomarkers for skeletal muscle toxicity, an unbiased metabolomic analysis was performed in rats treated with two distinct myotoxicants, cerivastatin (CER) and tetramethyl-p-phenylenediamine (TMPD). Skeletal muscle toxicity was induced in male Fischer 344 rats by administering CER or TMPD and monitored using established endpoints, such as increased plasma creatine kinase (CK) activity and histopathology, and a metabolomic analysis of skeletal muscle and plasma samples. Plasma CK levels in CER-treated rats were markedly elevated at Day 11; however, those in TMPD-treated rats showed a statistically significant decrease at $24 \mathrm{hr}$ after dosing. Light microscopy revealed that vacuolated or necrotic fibers were evident in all CER-treated rats on Day 11, and slightly vacuolated fibers were observed in TMPD-treated rats at 6 and $24 \mathrm{hr}$ after dosing. Metabolomic analysis of the rectus femoris indicated increases in 2-hydroxyglutarate (2HG) in CER-treated rats and hexanoylcarnitine in CER- and TMPD-treated rats. There were also increases in plasma 2HG in CER-treated rats on Days 8 and 11 and in TMPD-treated rats at $24 \mathrm{hr}$ after dosing and increases in plasma hexanoylcarnitine in CER-treated rats on Day 11 and in TMPD-treated rats at 6 and $24 \mathrm{hr}$ after dosing. These experiments demonstrated the potential of plasma $2 \mathrm{HG}$ and hexanoylcarnitine as specific and easily detectable biomarkers for skeletal muscle toxicity in rats and demonstrated the value of metabolomics for biomarker detection and identification in toxicological studies.
\end{abstract}

Key words: Biomarkers, Muscle, 2-Hydroxyglutarate, Hexanoylcarnitine, Metabolome, Rats

\section{INTRODUCTION}

Chemical- or drug-associated myotoxicity that ranges from myalgia to rhabdomyolysis is induced by treatment with antilipidemic drugs, hypocholesterolemic drugs, anesthetic agents, immunosuppressants, and other compounds (Oshima, 2011). Because rhabdomyolysis is life threatening, the detection of myotoxicity at the early phase is important for risk assessment and management. In the case of 3-hydroxy-3-methylglutaryl coenzyme A (HMG-CoA) reductase inhibitors (statins), fatal rhabdomyolysis is rare (Joy and Hegele, 2009), but among statins, cerivastatin induces rhabdomyolysis at a higher rate $(1.0 \%)$ (Kashani et al., 2006).

Early detection of skeletal muscle toxicity is important not only in nonclinical toxicity studies but also in clinical practice. Although creatine kinase (CK) and aspartate aminotransferase (AST) are conventional biomarkers for muscle impairment, their diagnostic utility is limited by their low sensitivity and specificity (Bohlmeyer et al., 1994; Sorichter et al., 1999). A previous study showed that an increase in CK levels after atorvastatin treatment was not associated with adverse effects on skeletal muscle function or the incidence of muscle pain (Ballard et al., 2013), suggesting that biomarkers with better sensitivity and specificity than those of $\mathrm{CK}$ are required for early muscle injury (Laaksonen, 2013). Several new biomarkers, including skeletal muscle troponin I (sTnI), myosin light chain 3 (Myl3), and fatty acid-binding protein 3 (FABP3), have been recommended for use in association

Correspondence: Fumitoshi Asai (E-mail: asai@azabu-u.ac.jp) 
with CK to monitor drug-induced skeletal muscle injury (Muntean et al., 2017). However, several drawbacks are associated with the use of these biomarkers, including their rapid clearance and alteration by renal dysfunction (Tonomura et al., 2012).

Metabolomics analysis, which involves the profiling of metabolites in biofluids, cells, and tissues, has been applied as a tool for biomarker discovery (Johnson et al., 2016). Compared to classical diagnostic approaches and conventional clinical biomarkers, metabolomics offers potential advantages owing to its sensitivity and specificity (Monteiro et al., 2013). Metabolomic approaches have been used to show changes in plasma acylcarnitines and urinary ethylmalonate, methylsuccinate, adipate, suberate, hexanoylglycine, sarcosine, dimethylglycine, isovalerylglycine, and 2-hydroxyglutarate $(2 \mathrm{HG})$ in cannabinoid-1 receptor antagonist-induced skeletal and cardiac myopathy in dogs (Tomlinson et al., 2012).

To identify new biomarkers that have better sensitivities than $\mathrm{CK}$, an unbiased metabolomic analysis of skeletal muscle and plasma using liquid chromatography/mass spectrometry (LC/MS) and gas chromatography (GC)/MS was performed on a drug- and chemical-induced skeletal muscle injury rat model in this study.

\section{MATERIALS AND METHODS}

\section{Chemicals and reagents}

Cerivastatin sodium (CER) was synthesized by Chemtec Labo., Inc. (Tokyo, Japan). TMPD was purchased from Sigma (St. Louis, MO, USA). CER was mixed with the powdered ingredients of a purified diet (CRF-1, Oriental Yeast Co., Ltd., Tokyo, Japan) at a concentration of 20 or $40 \mathrm{ppm}$. TMPD was suspended in a $0.5 \%$ methylcellulose solution (MC, \#400, Nacalai Tesque, Inc., Tokyo, Japan).

\section{Animals}

Male Fischer rats at 8 weeks of age were purchased from Charles River Japan, Inc. (Kanagawa, Japan). Animals were housed in stainless steel wire bracket cages in a room that was maintained at a temperature of 21 to $26^{\circ} \mathrm{C}$ with a relative humidity of 30 to $70 \%$, and lighting was maintained on a 12-hr light (7:00 to 19:00)/day cycle. All animals were allowed free access to water and a commercial rodent diet (CRF-1, Oriental Yeast Co., Ltd.). Rats were acclimatized for 1 week before use. All experimental procedures were performed in accordance with the guidelines of the Animal Care and Use Committee of Daiichi Sankyo Co., Ltd (Tokyo, Japan). The experimental protocols were approved by the Ethics Review Committee for
Animal Experimentation of Daiichi Sankyo Co., Ltd.

\section{Dosing}

Rats were fed a diet supplemented with 40 ppm CER or commercial diet only as a control. Blood samples for plasma CK measurement and muscle samples for histopathological and metabolomic analyses were obtained on Days 8 and 11 ( $n=5 \mathrm{rats} /$ dose group/time point). TMPD at a dose level of $0(0.5 \% \mathrm{MC})$ or $9 \mathrm{mg} / \mathrm{kg}$ was orally administered to the rats. The animals were sacrificed, and blood and muscle samples were collected 6 or $24 \mathrm{hr}$ after dosing ( $n=5 \mathrm{rats} /$ dose group/time point). The dose levels of each compound were selected on the basis of the pathological process of skeletal muscle injury reported previously (Obayashi et al., 2011; Pritt et al., 2008) and our preliminary study.

\section{Sample collection}

Blood samples were obtained from the abdominal aorta under isoflurane anesthesia at the respective sampling points and were treated with heparin for CK measurement and EDTA for metabolomic analysis. Plasma samples were kept at $-80^{\circ} \mathrm{C}$ until use. After blood collection, the animals were euthanized humanely by exsanguination, and skeletal muscles - soleus muscle, rectus femoris muscle, and tibialis anterior muscle-were excised for histopathological examination. The rectus femoris muscle was used for metabolomic analysis because the rectus femoris muscle was markedly damaged compared with the soleus and tibialis anterior muscles.

\section{Plasma CK analysis}

Plasma CK levels were measured using a standard commercial assay kit (L-Type CK, Wako Pure Chemical Industries, Ltd., Osaka, Japan) on an automated biochemical analyzer (7180, Hitachi High-Technologies Corporation, Tokyo, Japan) in accordance with the manufacturer's instructions.

\section{Histopathology}

Skeletal muscles (soleus, rectus femoris, and tibialis anterior muscles) from all control and treated rats were fixed in $10 \%$ buffered neutral formalin and embedded in paraffin. Muscle specimens were prepared by routine methods, stained with hematoxylin and eosin, and examined by light microscopy. Histopathological grading was employed to grade the pathological severity of lesions as within normal limits (-), slight $(+)$ or moderate $(++)$ (Mann et al., 2012). 
2HG and hexanoylcarnitine as biomarkers for skeletal muscle toxicity

\section{Metabolomic analysis}

For muscular metabolomic analysis, the rectus femoris muscle samples obtained on Day 11 (CER: 0 and $40 \mathrm{ppm}$ ) and at $24 \mathrm{hr}$ after dosing (TMPD: 0 and $9 \mathrm{mg} / \mathrm{kg}$ ) were used because histopathological changes in the rectus femoris muscle were evident at this time point. For plasma metabolomic analysis, plasma samples obtained at all sampling points were used. Metabolomic analysis was performed at Metabolon Inc. (Durham, NC, USA) with three independent platforms: ultrahigh-performance LC (ACQUITY UPLC, Waters, Milford, MA, USA) coupled to MS (Finnigan LTQ, Thermo Fisher Scientific Inc, Waltham, MA, USA) optimized for basic species or acidic species and GC/MS (Finnigan Trace DSQ, Thermo Fisher Scientific Inc.). Metabolites were extracted from each sample and analyzed in accordance with the method proposed by Evans et al. (2009). Metabolites were identified using known chemical structures by matching the ions' chromatographic retention indexes and mass spectral fragmentation signatures with reference library entries created from authentic standard metabolites following the identical analytical procedure used for the experimental samples (Dehaven et al., 2010).

\section{Data imputation and statistical analysis}

The missing values for a given metabolite were imputed as the observed minimum detection value on the assumption that they were below the limits of detection. For the convenience of data visualization, the raw area counts for each metabolite were rescaled by dividing each sample's value by the median value for the specific metabolite. Quantitative data are expressed as mean values \pm S.D. unless otherwise stated. For the metabolomic analysis data, the values of each metabolite are presented as a ratio of the value in the treated group to the mean value of the concurrent control group. All data were statistically analyzed using an F-test, followed by Student's t-test or Aspin-Welch's t-test between the treated groups and the concurrent control groups. These statistical analyses were performed with SAS ${ }^{\circledR}$ System Release 8.2 (SAS Institute Inc., Cary, NC, USA). A $P$ value $<0.05$ was considered statistically significant. Principal component analysis (PCA) of the identified biochemicals in the rectus femoris muscle with the highest fold-change values common to the CER- and TMPD-treated groups was performed using MetaboAnalyst 3.0 (Xia and Wishart, 2016).

\section{RESULTS}

\section{Plasma CK levels}

Plasma CK levels were determined in CER- and TMPD-treated rats in comparison with the respective vehicle control rats. There was no statistical difference in plasma CK levels between the vehicle control and CERtreated groups on Day 8 (Fig. 1A). However, the plasma CK levels in the CER-treated group were significantly $(P<0.05)$ higher than those in the control group on Day
$\mathbf{A}$

\section{Cerivastatin}

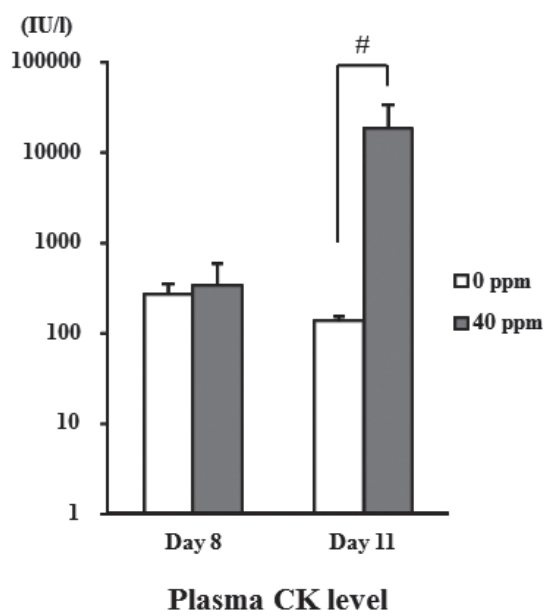

B

TMPD

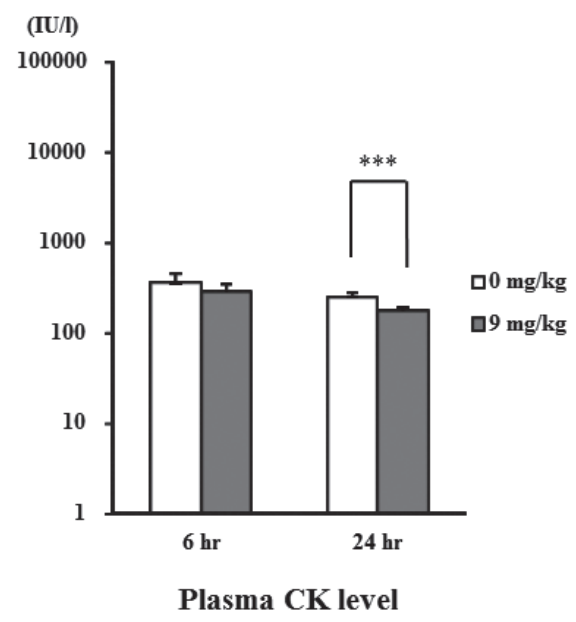

Fig. 1. Plasma creatine kinase (CK) levels in cerivastatin (CER)- and tetramethyl-p-phenylenediamine (TMPD)-treated rats. Plasma CK levels (mean \pm S.D., $n=5$ per group) of rats treated with 0 or $40 \mathrm{ppm}$ of CER on Days 8 and 11 (A) and 0 or $9 \mathrm{mg} / \mathrm{kg}$ of TMPD at 6 and $24 \mathrm{hr}$ after dosing (B). \# $P<0.05$ : compared with the control group by Aspin-Welch's $t$-test. $* * * P<0.001$ : compared with the control group by Student's $t$-test. 
$11(135 \pm 17 \mathrm{IU} / \mathrm{L}$ for control group, $18550 \pm 14258 \mathrm{IU} / \mathrm{L}$ for CER-treated group). In contrast, plasma CK levels in TMPD-treated rats were comparable to those in vehicle control rats at $6 \mathrm{hr}$ after dosing, and were significantly $(P<0.001)$ lower than those in the control rats at $24 \mathrm{hr}$ after dosing $(252 \pm 27 \mathrm{IU} / \mathrm{L}$ for control group, $177 \pm 13$ IU/L for TMPD-treated group, Fig. 1B).

\section{Histopathology}

To determine the effects of CER and TMPD, we evaluated the impact of myotoxicants on muscle degeneration on Days 8 and 11; these time points provided a useful comparison of the conditions before overt muscle damage (Day 8) and following muscle damage (Day 11). Light microscopy analysis revealed no abnormal findings for all of the muscle samples in the control groups (Table 1, Table 2, Fig. 2B, Fig. 2D) and the CER-treated rats necropsied on Day 8 (Table 1). Vacuolated or necrotic fibers were evident in the rectus femoris muscle in all CER-treated rats on Day 11 (Fig. 2A). In these rats, the tibialis anterior muscles were also mildly affected. Similar findings were also observed in 1 soleus muscle from 5 treated rats. For the TMPD-treated rats, vacuolated fibers were noted in all of the muscles examined at 6 and $24 \mathrm{hr}$ after dosing (Table 2, Fig. 2C).

\section{Metabolomic analysis of the rectus femoris muscle and plasma}

The biochemical profiles of the rectus femoris muscle in rats treated with CER on Day 11 or TMPD at $24 \mathrm{hr}$ after dosing were analyzed. This profiling identified 402 (279 named and 123 unnamed) biochemicals. Although unnamed biochemicals were found, we addressed only the named biochemicals in the present study. Of these identified biochemicals, 19 biochemicals were also among the 50 biochemicals with the highest fold change values in the CER- and TMPD-treated groups (Table 3). PCA of the biochemicals listed in Table 3 revealed a clear distinction between the CER- or TMPD-treated groups and the concurrent control group (Fig. 3).

Of these 19 biochemicals that were increased in the muscles in the CER- and TMPD-treated groups, plasma 2HG, 2-methylbutyroylcarnitine, uracil, and hexanoylcarnitine levels were also increased at the same point in time. However, 2-methylbutyroylcarnitine in the CERtreated group on Day 8 was significantly decreased, and uracil in the TMPD-treated group at $24 \mathrm{hr}$ was unchanged (Table 4). 2HG and hexanoylcarnitine represent the biochemicals that were elevated in both the muscle and in plasma samples of the groups given CER or TMPD (Fig. 4, Fig. 5).

Table 1. Histopathology of the cerivastatin-treated and control rats.

\begin{tabular}{|c|c|c|c|c|}
\hline \multicolumn{5}{|l|}{ Cerivastatin } \\
\hline \multirow{3}{*}{$\begin{array}{r}\text { Dose (ppm) } \\
\text { Day } \\
\text { No. of animals } \\
\end{array}$} & \multicolumn{2}{|c|}{0} & \multicolumn{2}{|c|}{40} \\
\hline & 8 & 11 & 8 & 11 \\
\hline & 5 & 5 & 5 & 5 \\
\hline \multicolumn{5}{|l|}{ Soleus muscle } \\
\hline Vacuolar degeneration, muscle fibers & - & - & - & $\begin{array}{c}1 \\
(+)\end{array}$ \\
\hline Necrosis, muscle fibers & - & - & - & $\begin{array}{l}1 \\
(+)\end{array}$ \\
\hline \multicolumn{5}{|l|}{ Rectus femoris muscle } \\
\hline Vacuolar degeneration, muscle fibers & - & - & - & $\begin{array}{c}5 \\
(++)\end{array}$ \\
\hline Necrosis, muscle fibers & - & - & - & $\begin{array}{c}5 \\
(++)\end{array}$ \\
\hline Cellular infiltration & - & - & - & $\begin{array}{c}3 \\
(+/++)\end{array}$ \\
\hline \multicolumn{5}{|l|}{ Tibialis anterior muscle } \\
\hline Vacuolar degeneration, muscle fibers & - & - & - & $\begin{array}{c}5 \\
(+)\end{array}$ \\
\hline Necrosis, muscle fibers & - & - & - & $\begin{array}{c}5 \\
(+)\end{array}$ \\
\hline
\end{tabular}

Grades: -, within normal limits; +, slight; +/++, slight to moderate; ++, moderate

The numbers of animals with pathological changes are listed. 
2HG and hexanoylcarnitine as biomarkers for skeletal muscle toxicity

Table 2. Histopathology of the tetramethyl-p-phenylenediamine-treated and control rats.

\begin{tabular}{|c|c|c|c|c|}
\hline \multicolumn{5}{|l|}{ Tetramethyl-p-phenylenediamine } \\
\hline \multirow{3}{*}{$\begin{array}{r}\text { Dose }(\mathrm{mg} / \mathrm{kg}) \\
\text { Time after dosing }(\mathrm{hr}) \\
\text { No. of animals }\end{array}$} & \multicolumn{2}{|c|}{0} & \multicolumn{2}{|c|}{9} \\
\hline & 6 & 24 & 6 & 24 \\
\hline & 5 & 5 & 5 & 5 \\
\hline \multicolumn{5}{|l|}{ Soleus muscle } \\
\hline Vacuolar degeneration, muscle fibers & - & - & $\begin{array}{c}5 \\
(+)\end{array}$ & $\begin{array}{c}5 \\
(+)\end{array}$ \\
\hline \multicolumn{5}{|l|}{ Rectus femoris muscle } \\
\hline Vacuolar degeneration, muscle fibers & - & - & $\begin{array}{c}5 \\
(+/++)\end{array}$ & $\begin{array}{c}5 \\
(++)\end{array}$ \\
\hline \multicolumn{5}{|l|}{ Tibialis anterior muscle } \\
\hline Vacuolar degeneration, muscle fibers & - & - & $\begin{array}{c}5 \\
(++)\end{array}$ & $\begin{array}{c}5 \\
(+)\end{array}$ \\
\hline Necrosis, muscle fibers & - & - & $\begin{array}{c}1 \\
(+)\end{array}$ & $\begin{array}{c}1 \\
(+)\end{array}$ \\
\hline
\end{tabular}

Grades: -, within normal limits; +, slight; +/++, slight to moderate; ++ , moderate

The numbers of animals with pathological changes are listed.

\section{DISCUSSION}

To identify novel biomarkers for skeletal muscle disorders, in the present study we examined the effects of two distinct myotoxicants, CER and TMPD, on the rat muscle and plasma metabolome. The present study showed that 1) CER- and TMPD-induced rat skeletal muscle injury models exhibit a distinct metabolic signature characterized by the elevation of $2 \mathrm{HG}$ and hexanoylcarnitine levels in muscle and plasma, 2) plasma $2 \mathrm{HG}$ increased prior to histopathological changes in skeletal muscle or the elevation of plasma CK in CER-treated rats, and 3) the increases in plasma $2 \mathrm{HG}$ and hexanoylcarnitine were accompanied by histopathological changes, although the plasma level of CK did not change at $6 \mathrm{hr}$ after dosing, and decreased at $24 \mathrm{hr}$ after dosing in the TMPD-treated rats. These results suggest that $2 \mathrm{HG}$ and hexanoylcarnitine may be novel plasma biomarkers for skeletal muscle damage.

We used CER and TMPD, which have different modes of toxicity, as myotoxicants to identify mechanism-independent biomarker candidates. Although the most compelling evidence suggests that statin myopathy is directly related to HMG-CoA reductase inhibition (Flint et al., 1997; Nishimoto et al., 2003; Johnson et al., 2004), the etiology and mechanism of statin-induced myopathy remain unclear. Mitochondria have been suggested to be direct targets of statin-induced toxicity both in vivo (De Pinieux et al., 1996; Schaefer et al., 2004; Obayashi et al., 2011) and in vitro (Nishimoto et al., 2003; Sirvent et al., 2005b; Kaufmann et al., 2006), although whether mitochondria are primary targets of statin-induced myotoxicity in rats is controversial (Waclawik et al., 1993;
Schaefer et al., 2004; Westwood et al., 2008). In this study, the type II fiber-predominant muscles, namely the rectus femoris and tibialis anterior muscles, were markedly damaged in CER-treated rats, whereas the type I fiber-predominant soleus muscle in the same rats was relatively preserved. These findings are consistent with several studies that have reported that statin-induced myotoxicity mainly targets type II fiber-predominant muscles (Reijneveld et al., 1996; Westwood et al., 2005, 2008). This fiber type-specific toxicity may be related to differences in mitochondrial content and energetics (Phillips and Haas, 2008). In contrast, fiber type-specific changes were not evident in the TMPD-treated rats. TMPD-induced myopathy is considered to be caused by the stable radical cations formed during autoxidation (Munday, 1988; Munday et al., 1990). Thus, although the skeletal muscle disorders caused by CER and TMPD result from different mechanisms, the biomarkers identified in the present study allowed for discrimination of these skeletal muscle injuries from the control, as determined via PCA (Fig. 3).

The administration of CER and TMPD at doses that induced skeletal muscle injury in rats resulted in increased levels of acylcarnitines in the rectus femoris muscle and plasma compared with those collected from control rats. It was reported that the off-target mechanism of myotoxicity accompanied by increased plasma levels of acylcarnitines in dogs that is induced by ibipinabant, which is a cannabinoid-1 receptor antagonist, involved the inhibition of mitochondrial ADP/ATP exchange (Schirris et al., 2015). It was suggested that increases in the plasma levels of acylcarnitines are due to impaired fatty acid oxidation in skeletal muscle (Tomlinson et al., 2012). It is well 
H. Obayashi et al.
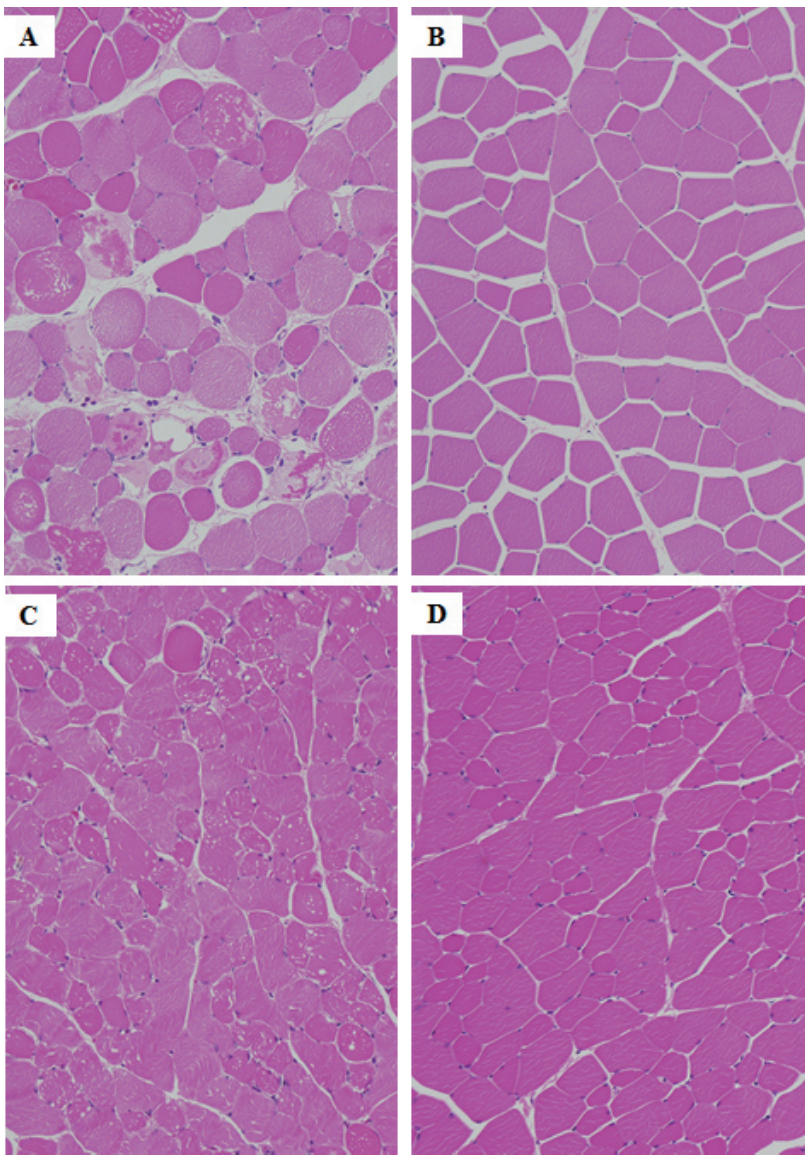

Fig. 2. Histopathological analysis of muscle fibers. The rectus femoris muscle from rats treated with 40 ppm CER (A) or 0 ppm CER (B) on Day 11, the tibialis anterior muscle from rats $6 \mathrm{hr}$ after treating with $9 \mathrm{mg} / \mathrm{kg}$ TMPD (C), and control vehicle (D). The following findings were noted: (A) marked variation in fiber size with moderately vacuolated and moderately necrotic fibers; (C) moderately vacuolated and slightly necrotic fibers; and (B) and (D) no abnormalities. Hematoxylin and eosin staining.

known that carnitine, which is essential for the transfer of long-chain fatty acids from the cytoplasm to the mitochondrial matrix for their oxidation (Bartlett and Eaton, 2004), plays an essential role in fatty acid metabolism (Bieber, 1988). Thus, increased muscular levels of hexanoylcarnitine and octanoylcarnitine were due to elevated fatty acid $\beta$-oxidation. It is known that a small fraction of acetyl-CoA generated from the $\beta$-oxidation of fatty acids is converted to the ketone bodies acetoacetate and 3-hydroxybutyrate. In the present study, the muscular levels of 3-hydroxybutyrate were increased, supporting an increase in $\beta$-oxidation activity.

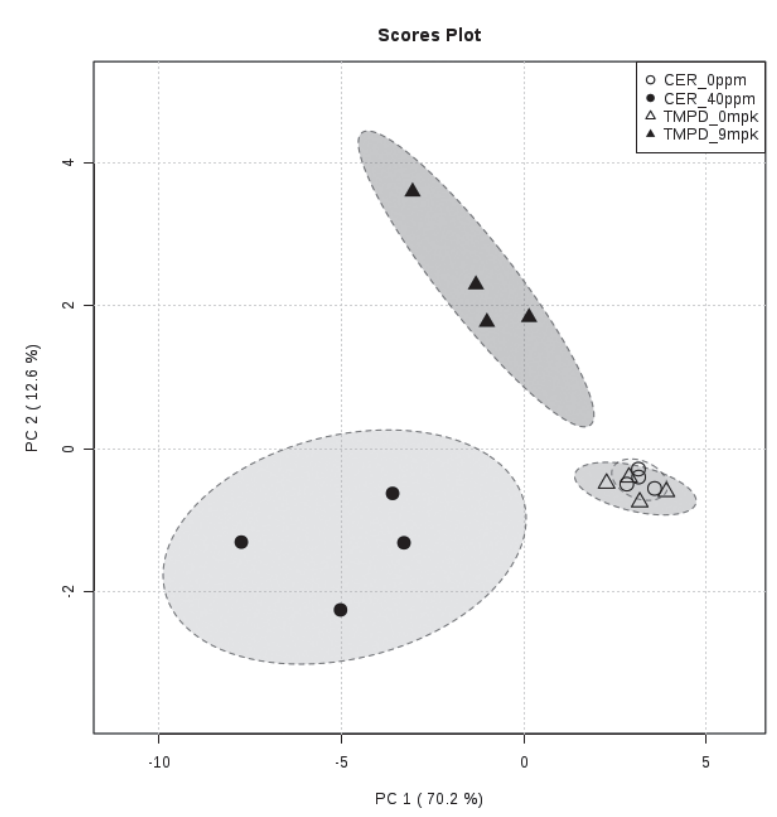

Fig. 3. Principal component analysis (PCA) score plots of the identified biochemicals in the rectus femoris muscle with the highest fold-change values common to the CER- and TMPD-treated groups. CER, cerivastatin $\mathrm{Na}$; TMPD, tetramethyl-p-phenylenediamine.

In this study, $2 \mathrm{HG}$ was elevated in both the muscles and plasma of each group given CER or TMPD, which may be due to impaired mitochondrial function. Recent studies have indicated that $2 \mathrm{HG}$ markedly impaired mitochondrial energy metabolism, especially, cytochrome c oxidase activity (Latini et al., 2005). 2HG is in redox-equilibrium with the tricarboxylic acid (TCA) cycle intermediate $\alpha$-ketoglutarate $(\alpha \mathrm{KG})$, and is produced from $\alpha \mathrm{KG}$ by malate dehydrogenase and lactate dehydrogenase $\mathrm{A}$ (Intlekofer et al., 2015). As $2 \mathrm{HG}$ is cleared via conversion to $\alpha \mathrm{KG}$ by $2 \mathrm{HG}$ dehydrogenase, which is a flavin adenine dinucleotide-dependent mitochondrial enzyme (Rzem et al., 2004), the plasma concentration of $2 \mathrm{HG}$ is maintained at a low level (less than $300 \mu \mathrm{M}$ ) in normal conditions (Dang et al., 2016). In hypoxic conditions, $2 \mathrm{HG}$ is elevated due to $2 \mathrm{HG}$ dehydrogenase down-regulation and then the increased amount of $2 \mathrm{HG}$ generates redox stress due to mitochondrial dysfunction by suppressing $2 \mathrm{HG}$ dehydrogenase. Mitochondrial abnormalities in statin-induced myotoxic lesions have been consistently observed for statins, including simvastatin, rosuvastatin, and pravastatin, in multiple species, including humans (Bergman et al., 2003; Gambelli et al., 2004; Seachrist et al., 2005; Sirvent et al., 2005a; Westwood et al., 2005, 2008). Mito- 
$2 \mathrm{HG}$ and hexanoylcarnitine as biomarkers for skeletal muscle toxicity

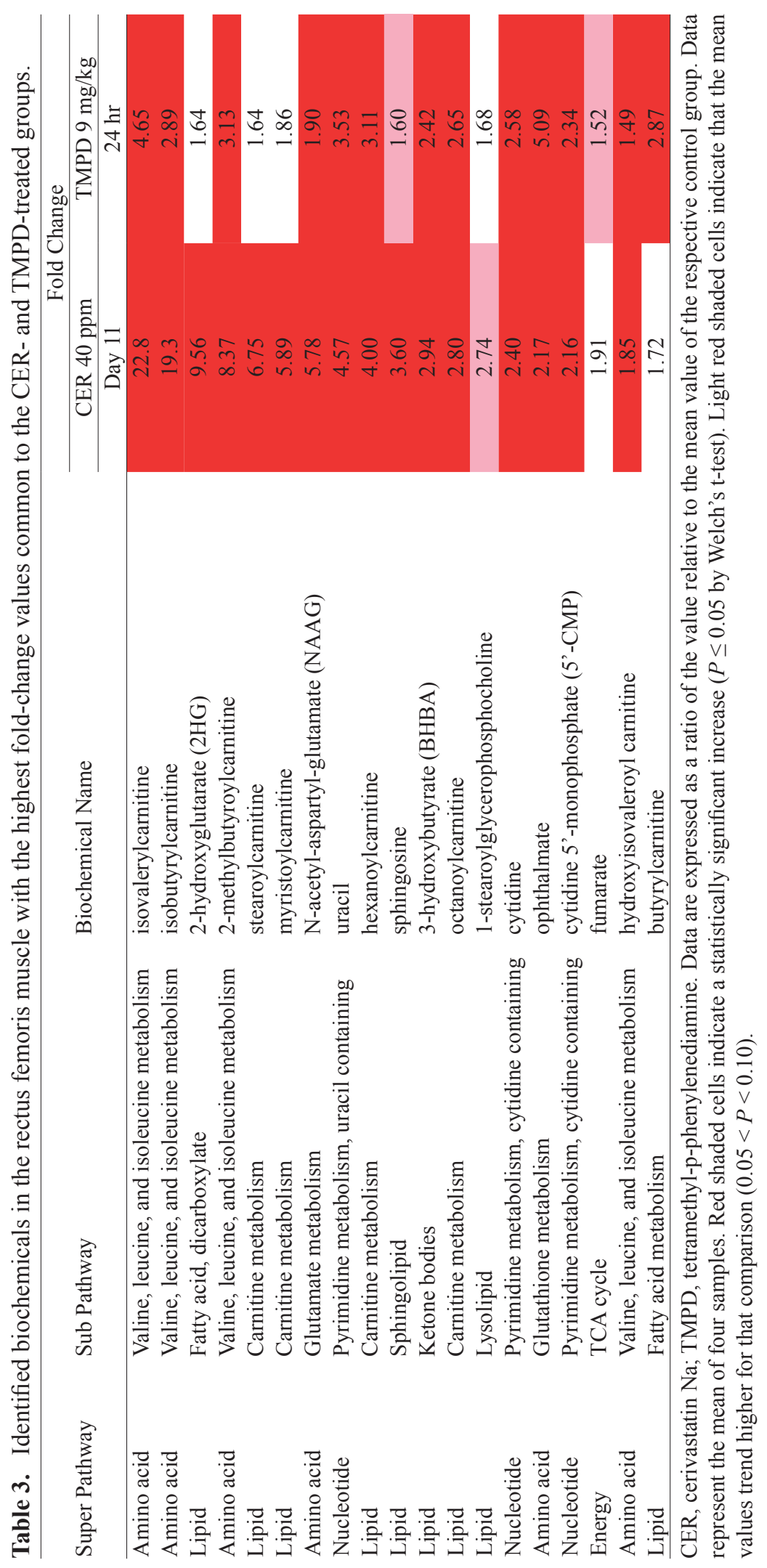


H. Obayashi et al.

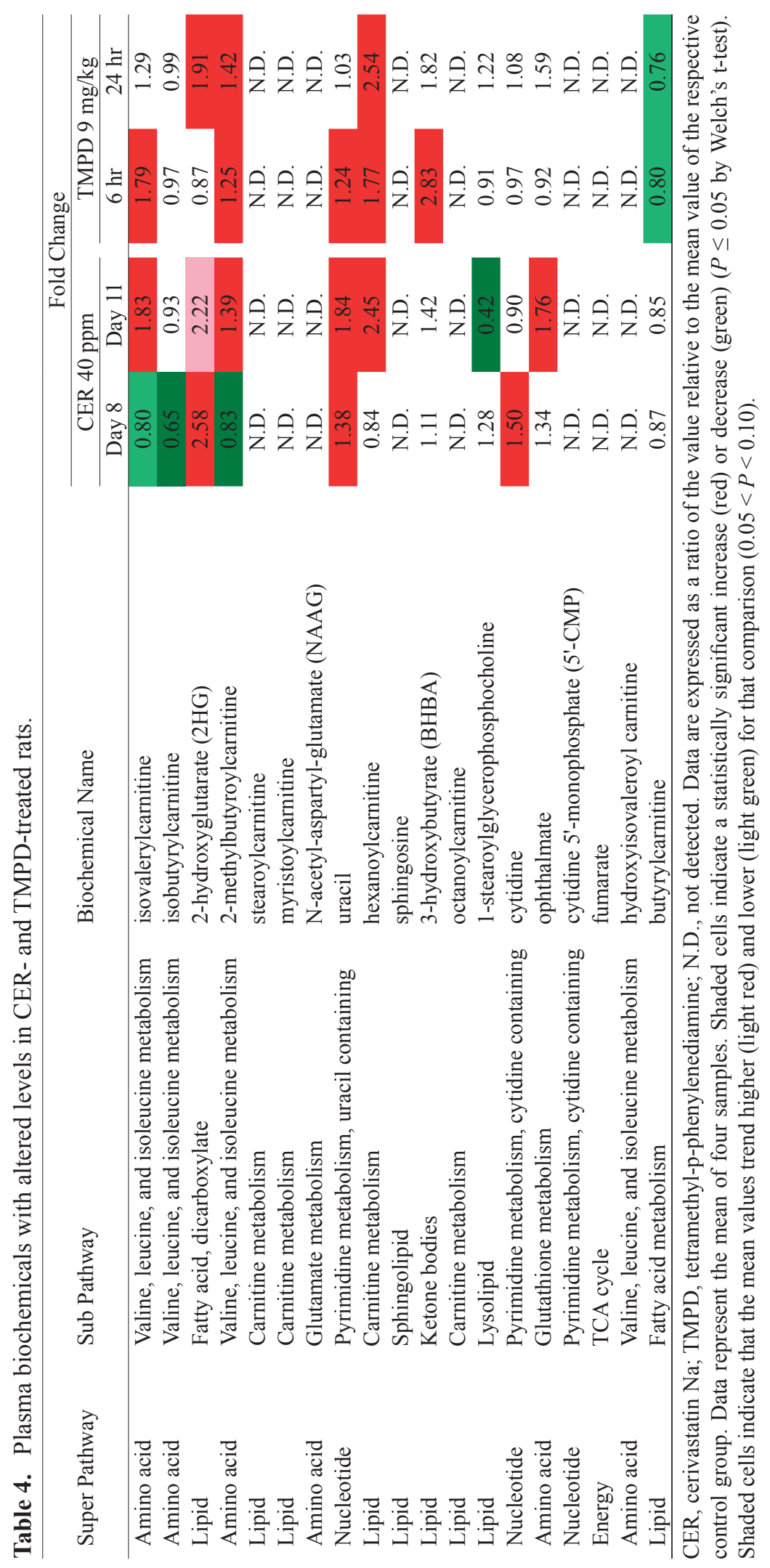

Vol. 42 No. 4 
2HG and hexanoylcarnitine as biomarkers for skeletal muscle toxicity
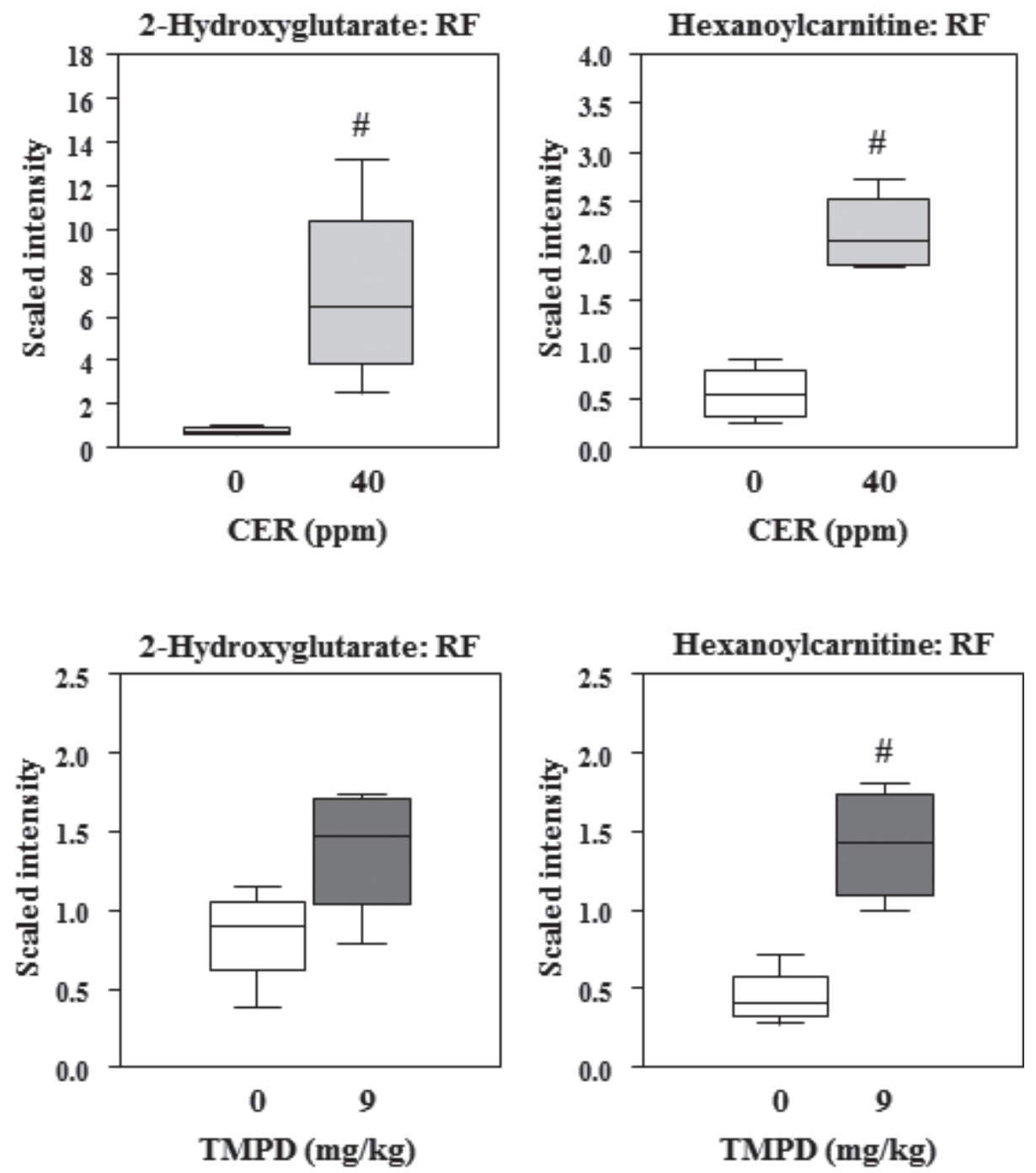

Fig. 4. Box-and-whisker plots of 2-hydroxyglutarate and hexanoylcarnitine in rectus femoris muscle samples. Metabolites in the rectus femoris muscle from rats treated with CER on Day 11 or TMPD at $24 \mathrm{hr}$ after dosing. The box represents the middle $50 \%$ of the distribution, and the upper and lower "whiskers" represent the entire spread of the data. The hyphen indicates the median. The name of each metabolite is indicated on the top of the plot. The y-axis is the median scaled value. $\# P \leq 0.05$ : Significantly different from the control group by Welch's t-test. CER, cerivastatin Na; TMPD, tetramethyl-pphenylenediamine; RF, rectus femoris muscle.

chondrial oxidation of TMPD is considered important in the initiation of its myotoxic action because muscle is particularly rich in mitochondria and is largely dependent on aerobic metabolism to meet its energy requirements (Munday et al., 1990). Although it is unclear whether CER and TMPD increase the synthesis or decrease the degradation of $2 \mathrm{HG}$, it is suggested that the accumulation of $2 \mathrm{HG}$ diverts electrons from the TCA cycle and affects the electron transport mechanism, thus contributing to CER- and TMPD-induced muscular dysfunction.
Our present data, which show that the levels of $2 \mathrm{HG}$ and hexanoylcarnitine were increased in plasma and muscle tissues, suggest that CER- and TMPD-induced skeletal muscular injury can be monitored in the plasma, which would be useful in routine animal studies. In addition, $2 \mathrm{HG}$ and hexanoylcarnitine may be potential biomarkers for muscle toxicity in dogs, because Tomlinson et al. (2012) reported that 2HG and hexanoylcarnitine were increased in dogs with skeletal and cardiac myopathies induced by cannabinoid-1 receptor antagonist. 

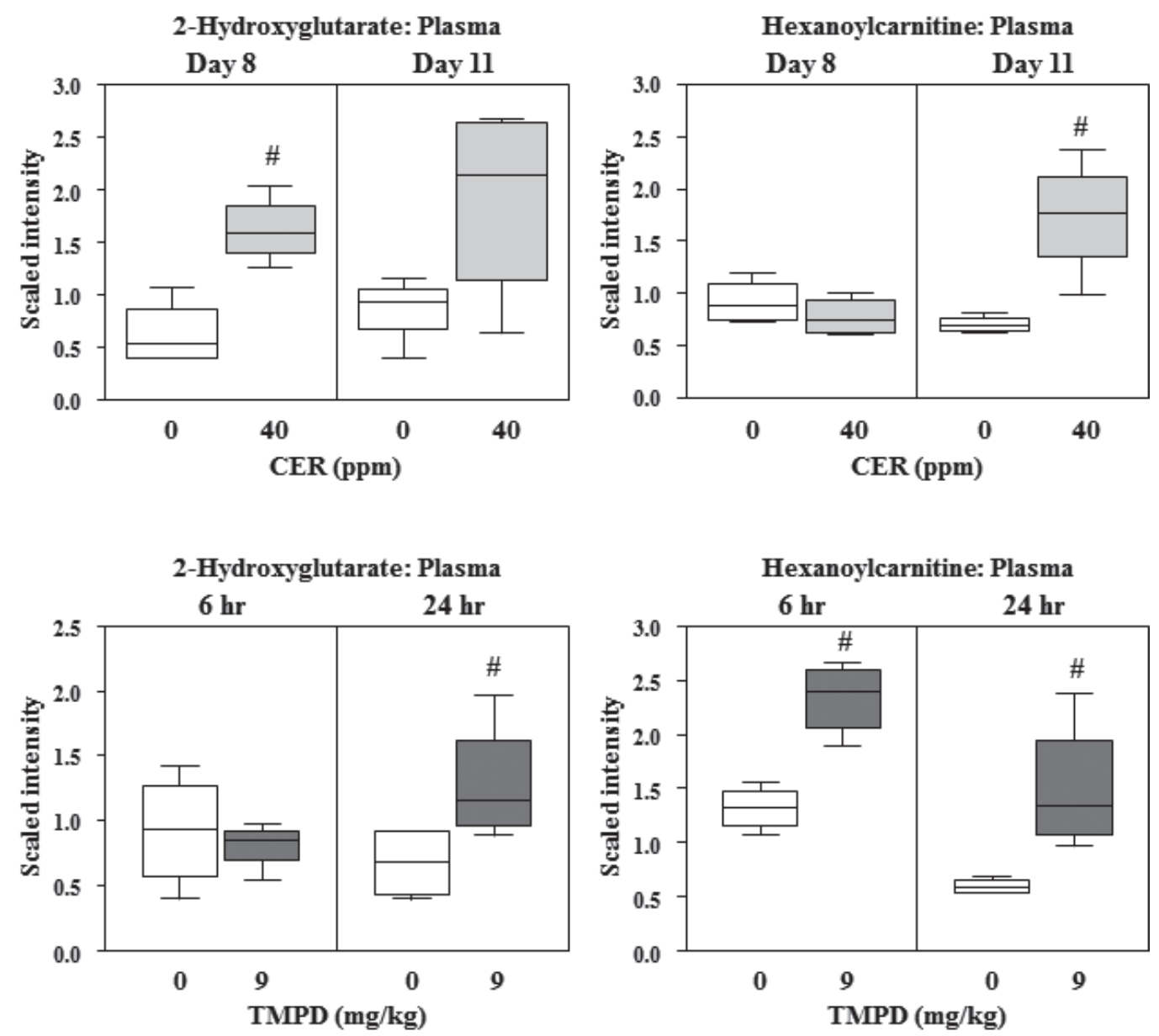

Fig. 5. Box-and-whisker plots of 2-hydroxyglutarate and hexanoylcarnitine in plasma samples. The box represents the middle $50 \%$ of the distribution, and the upper and lower "whiskers" represent the entire spread of the data. The hyphen indicates the median. The name of each metabolite is indicated on the top of the plot. The y-axis is the median scaled value. \# $P \leq 0.05$ : Significantly different from the control group by Welch's t-test. CER, cerivastatin Na; TMPD, tetramethyl-p-phenylenediamine.

The plasma level of $2 \mathrm{HG}$ in CER-treated rats increased on Day 8, prior to the development of histopathological changes and the increase in the plasma level of CK. Moreover, the increased plasma levels of $2 \mathrm{HG}$ and hexanoylcarnitine were accompanied by histopathological changes, although the plasma level of CK did not change in TMPD-treated rats. Collectively, these results suggest that plasma $2 \mathrm{HG}$ and hexanoylcarnitine are more sensitive than CK for skeletal muscle injury at the early stage.

By contrast, the plasma levels of $2 \mathrm{HG}$ and CK did not increase at $6 \mathrm{hr}$ after administering TMPD despite the presence of histopathological changes. The reason for this discrepancy between the plasma $2 \mathrm{HG}$ and CK levels and histopathological changes in TMPD-treated rats is not clear, but it may be associated with the severity of skeletal muscle injury. It is known that mild muscle lesions are mainly composed of cytoplasmic vacuolation, and under these pathological conditions, the plasma levels of $2 \mathrm{HG}$ and CK may not reflect their levels in muscle. Indeed, an early study suggested that a lack of elevated plasma CK does not rule out statin-associated structural muscle injury characterized by vacuolation of the T-tubular system (Mohaupt et al., 2009). In contrast, the plasma level of CK increased more than 100 times compared with that in control rats, whereas the plasma levels of $2 \mathrm{HG}$ and hexanoylcarnitine increased approximately 2.5 times. Thus, the plasma level of CK may be more sensitive than the plasma levels of $2 \mathrm{HG}$ and hexanoylcarnitine for relative- 
2HG and hexanoylcarnitine as biomarkers for skeletal muscle toxicity

ly severe myopathy such as that seen in the CER-treated rats on Day 11.

As far as we know, this is the first report showing an increase in plasma levels of $2 \mathrm{HG}$ and hexanoylcarnitine with chemical-induced skeletal muscle injury in rats. However, our study has several limitations. We did not evaluate the organ or species specificity of each candidate biomarker for muscle injury. Therefore, additional study will be needed to assess these candidate biomarkers in other models of skeletal muscle toxicity and in other general models, including those of hepatic, renal, or cardiac toxicity, in rats and other species. Furthermore, a comparison of these candidate biomarkers with other biomarkers for skeletal muscle injury except for total CK, such as CK-MM, sTnI, Myl3 and FABP3, would provide valuable knowledge. Finally, a relative quantitative analysis of metabolites was conducted in the present study. To confirm the use of the candidate biomarkers, validation of the assay system and accumulation of background data are need.

In conclusion, the present study demonstrated the potential of plasma $2 \mathrm{HG}$ and hexanoylcarnitine as specific and easily detectable biomarkers for skeletal muscle toxicity in rats and demonstrated the value of metabolomics for biomarker detection and identification in toxicological studies.

\section{ACKNOWLEDGMENTS}

We would like thank Sanae Takada and Tomohiro Watanabe for their technical assistance.

Conflict of interest---- The authors declare that there is no conflict of interest.

\section{REFERENCES}

Ballard, K.D., Parker, B.A., Capizzi, J.A., Grimaldi, A.S., Clarkson, P.M., Cole, S.M., Keadle, J., Chipkin, S., Pescatello, L.S., Simpson, K., White, C.M. and Thompson, P.D. (2013): Increases in creatine kinase with atorvastatin treatment are not associated with decreases in muscular performance. Atherosclerosis, 230, 121-124.

Bartlett, K. and Eaton, S. (2004): Mitochondrial beta-oxidation. Eur. J. Biochem., 271, 462-469.

Bergman, M., Salman, H., Djaldetti, M., Alexandrova, S., Punsky, I. and Bessler, H. (2003): Ultrastructure of mouse striated muscle fibers following pravastatin administration. J. Muscle Res. Cell Motil., 24, 417-420.

Bieber, L.L. (1988): Carnitine. Annu. Rev. Biochem., 57, 261-283.

Bohlmeyer, T.J., Wu, A.H. and Perryman, M.B. (1994): Evaluation of laboratory tests as a guide to diagnosis and therapy of myositis. Rheum. Dis. Clin. North Am., 20, 845-856.

Dang, L., Yen, K. and Attar, E.C. (2016): IDH mutations in cancer and progress toward development of targeted therapeutics. Ann. Oncol., 27, 599-608.

De Pinieux, G., Chariot, P., Ammi-Saïd, M., Louarn, F., Lejonc, J.L., Astier, A., Jacotot, B. and Gherardi, R. (1996): Lipid-lowering drugs and mitochondrial function: effects of HMG-CoA reductase inhibitors on serum ubiquinone and blood lactate/pyruvate ratio. Br. J. Clin. Pharmacol., 42, 333-337.

Dehaven, C.D., Evans, A.M., Dai, H. and Lawton, K.A. (2010): Organization of GC/MS and LC/MS metabolomics data into chemical libraries. J. Cheminform., 2, 9.

Evans, A.M., DeHaven, C.D., Barrett, T., Mitchell, M. and Milgram, E. (2009): Integrated, nontargeted ultrahigh performance liquid chromatography/electrospray ionization tandem mass spectrometry platform for the identification and relative quantification of the small-molecule complement of biological systems. Anal. Chem., 81, 6656-6667.

Flint, O.P., Masters, B.A., Gregg, R.E. and Durham, S.K. (1997): HMG CoA reductase inhibitor-induced myotoxicity: pravastatin and lovastatin inhibit the geranylgeranylation of low-molecular-weight proteins in neonatal rat muscle cell culture. Toxicol. Appl. Pharmacol., 145, 99-110.

Gambelli, S., Dotti, M.T., Malandrini, A., Mondelli, M., Stromillo, M.L., Gaudiano, C. and Federico, A. (2004): Mitochondrial alterations in muscle biopsies of patients on statin therapy. J. Submicrosc. Cytol. Pathol., 36, 85-89.

Intlekofer, A.M., Dematteo, R.G., Venneti, S., Finley, L.W., Lu, C., Judkins, A.R., Rustenburg, A.S., Grinaway, P.B., Chodera, J.D., Cross, J.R. and Thompson, C.B. (2015): Hypoxia Induces Production of L-2-Hydroxyglutarate. Cell Metab, 22, 304-311.

Johnson, C.H., Ivanisevic, J. and Siuzdak, G. (2016): Metabolomics: beyond biomarkers and towards mechanisms. Nat. Rev. Mol. Cell Biol., 17, 451-459.

Johnson, T.E., Zhang, X., Bleicher, K.B., Dysart, G., Loughlin, A.F., Schaefer, W.H. and Umbenhauer, D.R. (2004): Statins induce apoptosis in rat and human myotube cultures by inhibiting protein geranylgeranylation but not ubiquinone. Toxicol. Appl. Pharmacol., 200, 237-250.

Joy, T.R. and Hegele, R.A. (2009): Narrative review: statin-related myopathy. Ann. Intern. Med., 150, 858-868.

Kashani, A., Phillips, C.O., Foody, J.M., Wang, Y., Mangalmurti, S., Ko, D.T. and Krumholz, H.M. (2006): Risks associated with statin therapy: a systematic overview of randomized clinical trials. Circulation, 114, 2788-2797.

Kaufmann, P., Török, M., Zahno, A., Waldhauser, K.M., Brecht, K. and Krähenbühl, S. (2006): Toxicity of statins on rat skeletal muscle mitochondria. Cell. Mol. Life Sci., 63, 2415-2425.

Laaksonen, R. (2013): STOMPing forward: statins, muscle complaints and CK. Atherosclerosis, 230, 256-257.

Latini, A., da Silva, C.G., Ferreira, G.C., Schuck, P.F., Scussiato, K., Sarkis, J.J., Dutra Filho, C.S., Wyse, A.T., Wannmacher, C.M. and Wajner, M. (2005): Mitochondrial energy metabolism is markedly impaired by D-2-hydroxyglutaric acid in rat tissues. Mol. Genet. Metab., 86, 188-199.

Mann, P.C., Vahle, J., Keenan, C.M., Baker, J.F., Bradley, A.E., Goodman, D.G., Harada, T., Herbert, R., Kaufmann, W., Kellner, R., Nolte, T., Rittinghausen, S. and Tanaka, T. (2012): International harmonization of toxicologic pathology nomenclature: an overview and review of basic principles. Toxicol. Pathol., 40, 7S-13S.

Mohaupt, M.G., Karas, R.H., Babiychuk, E.B., Sanchez-Freire, V., Monastyrskaya, K., Iyer, L., Hoppeler, H., Breil, F. and Draeger, A. (2009): Association between statin-associated myopathy and 
skeletal muscle damage. CMAJ, 181, E11-18.

Monteiro, M.S., Carvalho, M., Bastos, M.L. and Guedes de Pinho, P. (2013): Metabolomics analysis for biomarker discovery: advances and challenges. Curr. Med. Chem., 20, 257-271.

Munday, R. (1988): Generation of superoxide radical, hydrogen peroxide and hydroxyl radical during the autoxidation of $\mathrm{N}, \mathrm{N}, \mathrm{N}^{\prime}, \mathrm{N}^{\prime}-$ tetramethyl-p-phenylenediamine. Chem. Biol. Interact., 65, 133143.

Munday, R., Manns, E., Fowke, E.A. and Hoggard, G.K. (1990): Structure-activity relationships in the myotoxicity of ring-methylated p-phenylenediamines in rats and correlation with autoxidation rates in vitro. Chem. Biol. Interact., 76, 31-45.

Muntean, D.M., Thompson, P.D., Catapano, A.L., Stasiolek, M., Fabis, J., Muntner, P., Serban, M.C. and Banach, M. (2017): Statin-associated myopathy and the quest for biomarkers: can we effectively predict statin-associated muscle symptoms? Drug Discov. Today, 22, 85-96.

Nishimoto, T., Tozawa, R., Amano, Y., Wada, T., Imura, Y. and Sugiyama, Y. (2003): Comparing myotoxic effects of squalene synthase inhibitor, T-91485, and 3-hydroxy-3-methylglutaryl coenzyme A (HMG-CoA) reductase inhibitors in human myocytes. Biochem. Pharmacol., 66, 2133-2139.

Obayashi, H., Nezu, Y., Yokota, H., Kiyosawa, N., Mori, K., Maeda, N., Tani, Y., Manabe, S. and Sanbuissho, A. (2011): Cerivastatin induces type-I fiber-, not type-II fiber-, predominant muscular toxicity in the young male F344 rats. J. Toxicol. Sci., 36, 445-452.

Oshima, Y. (2011): Characteristics of drug-associated rhabdomyolysis: analysis of 8,610 cases reported to the U.S. Food and Drug Administration. Intern. Med., 50, 845-853.

Phillips, P.S. and Haas, R.H. (2008): Statin myopathy as a metabolic muscle disease. Expert Rev. Cardiovasc. Ther., 6, 971-978.

Pritt, M.L., Hall, D.G., Recknor, J., Credille, K.M., Brown, D.D., Yumibe, N.P., Schultze, A.E. and Watson, D.E. (2008): Fabp3 as a biomarker of skeletal muscle toxicity in the rat: comparison with conventional biomarkers. Toxicol. Sci., 103, 382-396.

Reijneveld, J.C., Koot, R.W., Bredman, J.J., Joles, J.A. and Bär, P.R. (1996): Differential effects of 3-hydroxy-3-methylglutarylcoenzyme A reductase inhibitors on the development of myopathy in young rats. Pediatr. Res., 39, 1028-1035.

Rzem, R., Veiga-da-Cunha, M., Noël, G., Goffette, S., Nassogne, M.C., Tabarki, B., Schöller, C., Marquardt, T., Vikkula, M. and Van Schaftingen, E. (2004): A gene encoding a putative FADdependent L-2-hydroxyglutarate dehydrogenase is mutated in L-2-hydroxyglutaric aciduria. Proc. Natl. Acad. Sci. USA, 101, 16849-16854.

Schaefer, W.H., Lawrence, J.W., Loughlin, A.F., Stoffregen, D.A., Mixson, L.A., Dean, D.C., Raab, C.E., Yu, N.X., Lankas, G.R. and Frederick, C.B. (2004): Evaluation of ubiquinone concen- tration and mitochondrial function relative to cerivastatin-induced skeletal myopathy in rats. Toxicol. Appl. Pharmacol., 194, 10-23.

Schirris, T.J., Ritschel, T., Herma Renkema, G., Willems, P.H., Smeitink, J.A. and Russel, F.G. (2015): Mitochondrial ADP/ATP exchange inhibition: a novel off-target mechanism underlying ibipinabant-induced myotoxicity. Sci. Rep., 5, 14533.

Seachrist, J.L., Loi, C.M., Evans, M.G., Criswell, K.A. and Rothwell, C.E. (2005): Roles of exercise and pharmacokinetics in cerivastatin-induced skeletal muscle toxicity. Toxicol. Sci., $\mathbf{8 8}, 551-561$.

Sirvent, P., Bordenave, S., Vermaelen, M., Roels, B., Vassort, G., Mercier, J., Raynaud, E. and Lacampagne, A. (2005a): Simvastatin induces impairment in skeletal muscle while heart is protected. Biochem. Biophys. Res. Commun., 338, 1426-1434.

Sirvent, P., Mercier, J., Vassort, G. and Lacampagne, A. (2005b): Simvastatin triggers mitochondria-induced $\mathrm{Ca} 2+$ signaling alteration in skeletal muscle. Biochem. Biophys. Res. Commun., 329, 1067-1075.

Sorichter, S., Puschendorf, B. and Mair, J. (1999): Skeletal muscle injury induced by eccentric muscle action: muscle proteins as markers of muscle fiber injury. Exerc. Immunol. Rev., 5, 5-21.

Tomlinson, L., Tirmenstein, M.A., Janovitz, E.B., Aranibar, N., Ott, K.H., Kozlosky, J.C., Patrone, L.M., Achanzar, W.E., Augustine, K.A., Brannen, K.C., Carlson, K.E., Charlap, J.H., Dubrow, K.M., Kang, L., Rosini, L.T., Panzica-Kelly, J.M., Flint, O.P., Moulin, F.J., Megill, J.R., Zhang, H., Bennett, M.J. and Horvath, J.J. (2012): Cannabinoid receptor antagonist-induced striated muscle toxicity and ethylmalonic-adipic aciduria in beagle dogs. Toxicol. Sci., 129, 268-279.

Tonomura, Y., Matsushima, S., Kashiwagi, E., Fujisawa, K., Takagi, S., Nishimura, Y., Fukushima, R., Torii, M. and Matsubara, M. (2012): Biomarker panel of cardiac and skeletal muscle troponins, fatty acid binding protein 3 and myosin light chain 3 for the accurate diagnosis of cardiotoxicity and musculoskeletal toxicity in rats. Toxicology, 302, 179-189.

Waclawik, A.J., Lindal, S. and Engel, A.G. (1993): Experimental lovastatin myopathy. J. Neuropathol. Exp. Neurol., 52, 542-549.

Westwood, F.R., Bigley, A., Randall, K., Marsden, A.M. and Scott, R.C. (2005): Statin-induced muscle necrosis in the rat: distribution, development, and fibre selectivity. Toxicol. Pathol., 33, 246-257.

Westwood, F.R., Scott, R.C., Marsden, A.M., Bigley, A. and Randall, K. (2008): Rosuvastatin: characterization of induced myopathy in the rat. Toxicol. Pathol., 36, 345-352.

Xia, J. and Wishart, D.S. (2016): Using MetaboAnalyst 3.0 for Comprehensive Metabolomics Data Analysis. Current protocols in bioinformatics, 55, 14.10.1-14.10.91. 